\title{
Fidelity of polarization and spatial information recovery using a fiber-coupled phase-conjugate mirror
}

\author{
Yasuo Tomita, Ram Yahalom, and Amnon Yariv \\ California Institute of Technology, Pasadena, California 91125
}

Received April 28, 1987; accepted September 11, 1987

\begin{abstract}
We report the investigation of polarization and spatial information recovery using a fiber-coupled phase-conjugate mirror. A marked dependence of the phase-conjugate output on the input-beam conditions is experimentally observed. A theoretical model for these phenomena is also discussed.
\end{abstract}

We recently reported on polarization-preserving (true) phase conjugation, which is based on the use of a (multi)mode scrambling fiber that is terminated by a conventional passive phase-conjugate mirror (PCM) ${ }^{1,2}$ and on its new applications. ${ }^{3,4}$ The potential applications of such a device in sensor and optical image processing is considerable, so we undertook a study to determine the quality and parametric dependence of the polarization and spatial information recovery of the fiber-PCM combination.

The experimental arrangement is shown in Fig. 1. A multilongitudinal-mode argon-ion laser $(\lambda=514.5$ $\mathrm{nm}$ ) was used as a light source. The $x$-polarized input beam $\mathbf{E}^{(1)}$ was focused into the multimode gradedindex fiber (numerical aperture N.A.fiber $=0.29 ; 5 \mathrm{~m}$ long) through a lens (L1). The input-beam diameter $\phi$ (and therefore the input-beam N.A., which we will also designate by $\phi$ ) was controlled by an aperture (AP1). The microscope objective (L2) of 0.40 N.A. at the output end of the fiber was used to collect all the modes emitted from the fiber. $\mathrm{A} \mathrm{BaTiO}_{3}$ crystal was used as a self-pumped phase conjugate $\mathrm{PCM}^{5}$ for the $x$-polarized incident beam of $1 \mathrm{~mW}$. The transmissivity of the fiber $\left(\left|\mathbf{E}^{(2)} / \mathbf{E}^{(1)}\right| 2\right)$ was about $60 \%$, and the phase-conjugate reflectivity $\left(\equiv\left|\mathbf{E}^{(3)} / \mathbf{E}_{x}^{(2)}\right|^{2}\right)$ at the crystal was $30 \%$, independently of $\phi$. The phase-conjugate beam $\mathbf{E}^{(4)}$ from the input end of the fiber is reflected by the nonpolarizing beam splitter (BS1). Its power and its spatial structure were recorded by a detector (D4) and a TV camera, respectively. It was ascertained by means of the $\lambda / 4$ plate and the analyzer (A) that BS1 has no effect on the ellipsometric measurement of the Stokes parameters $\left(s_{0}, s_{1}, s_{2}, s_{3}\right)$ and therefore on the degree of polarization $P\left[\equiv\left(s_{1}{ }^{2}+s_{2}{ }^{2}+\right.\right.$ $\left.s_{3}^{2}\right)^{1 / 2} / s_{0}$ ] (Ref. 6) for $\mathbf{E}^{(4)}$. An aperture (AP2) with a diameter $\Phi$ was placed in front of the $\lambda / 4$ plate to limit the detecting area for $\mathbf{E}^{(4)}$.

Figure 2 shows the results of a measurement of the degree of polarization recovery $p \equiv s_{1} / s_{0}=\left(P_{x}-P_{y}\right) /$ $\left(P_{x}+P_{y}\right)\left(P_{x}\right.$ and $P_{y}$ are the power of $x$ - and $y$-polarized components of $\mathbf{E}^{(4)}$ ) and the degree of polarization $P$ defined above, together with the reflectivity $R \equiv$ $\left|\mathbf{E}_{x}^{(4)} / \mathbf{E}^{(1)}\right| 2$, as a function of the fraction $\left(\phi / \phi_{0}\right)^{2}$ of the number of fiber modes that are excited initially $\left(\phi_{0}\right.$ is the input beam diameter, which corresponds to N.A.fiber) for the linearly $x$-polarized input $(p, P>0.99)$. The diameter $\Phi$ of AP2 was set to be $\Phi=\phi$ for all $\phi$ 's. All the data were obtained within uncertainties of $\pm 10 \%$. It is shown that for very small $\phi$ (i.e., when the N.A. of the input beam is very small compared with N.A. fiber), $p$ is almost unity, i.e., true phase conjugation of the input beam $\mathbf{E}^{(1)}$ is possible, whereas $R$ is low. On the other hand, $p$ decreases appreciably with the increase of $\phi$, accompanied by an increase in $R$. It is also seen that $p \approx P$ for all $\phi$ 's, indicating that the polarized part of the phase-conjugate beam is almost $x$-polarized and that the rest of the phase-conjugate beam is completely depolarized. Thus the power in the depolarized component of the reflected beam increases with the increase of $\phi$, making the total $R$ increase but $p$ and $P$ decrease. We also measured $p$ and $P$ as a function of $\Phi / \phi$ for a small N.A. $\left[\left(\phi / \phi_{0}\right)^{2}=0.005\right]$ and a large N.A. $\left[\left(\phi / \phi_{0}\right)^{2}=0.69\right]$. It was found that for the small N.A. $p$ starts decreasing when $\Phi / \phi$ exceeds unity, while for the large N.A. $p$ is nearly constant regardless of $\Phi / \phi$.

The above data are consistent with a model according to which the nearly $50 \%$ of the reflected power that is not the power of the (polarized) true phase-conjugate component but is that of the depolarized component is distributed essentially uniformly over all the fiber modes, thus occupying $\phi_{0}$ independently of $\phi$. This depolarized component will be referred to as noise. If the input beam occupies $\phi \ll \phi_{0}$, the full recovery of the true phase-conjugate signal is accomplished with an acceptance-beam diameter $\Phi=\phi$, re-

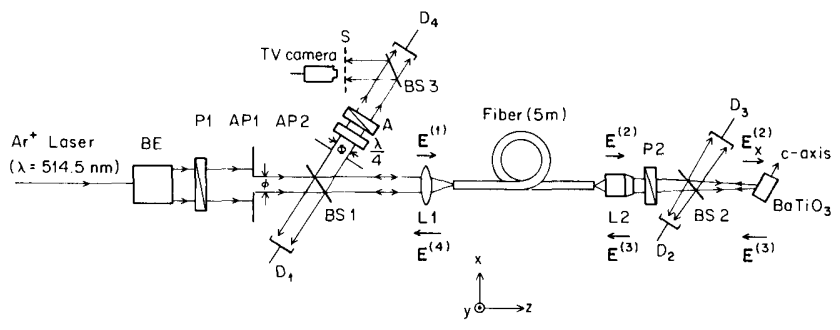

Fig. 1. Experimental arrangement. BE, beam expander; $\mathrm{P} 1, \mathrm{P} 2$, polarizers to guarantee the $x$-polarized input to the fiber and the crystal, respectively; D1, D2, D3, detectors for measuring the power of the beams $\mathbf{E}^{(1)}, \mathbf{E}_{x}^{(2)}$, and $\mathbf{E}^{(3)}$, respectively. 


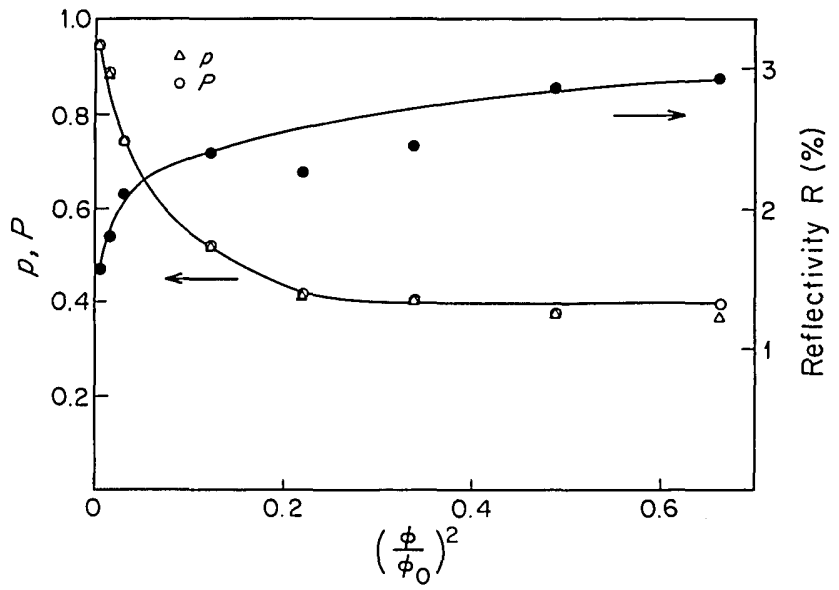

Fig. 2. Degree of polarization recovery $p$ and degree of polarization $P$ together with reflectivity $R$ as a function of $\left(\phi / \phi_{0}\right)^{2}$ for the linearly $x$-polarized input: $p(\Delta), P(0)$, and $R(\bullet)$. The diameter $\Phi$ of AP2 is identical to $\phi$.

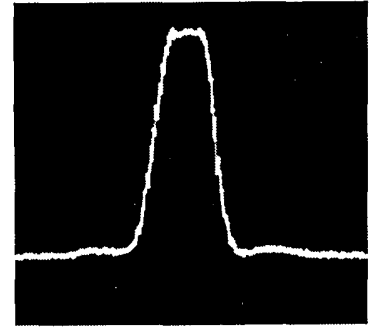

(a)
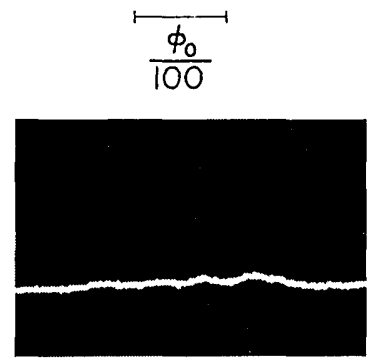

(b)

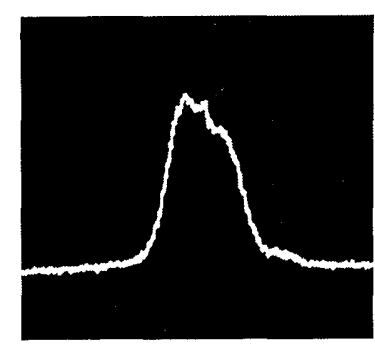

(c)
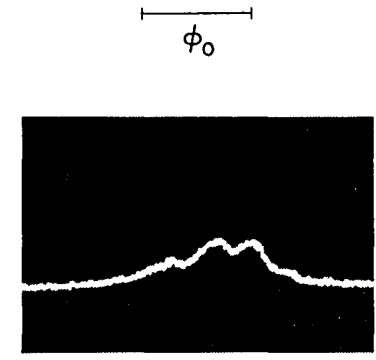

(d)
Fig. 3. Intensity distributions of phase-conjugate output beams for the linearly $x$-polarized input: (a) $x$-polarized component (signal + noise) and (b) $y$-polarized component (noise) for $\left(\phi / \phi_{0}\right)^{2}=0.005$; (c) $x$-polarized component (signal + noise) and (d) $y$-polarized component (noise) for $\left(\phi / \phi_{0}\right)^{2}=$ 0.69 .

sulting in a rejection of most of the noise power since only a fraction $\left(\phi / \phi_{0}\right)^{2} \ll 1$ of the noise power is contained within $\phi$ occupied by the signal. This leads to $p$ $\geq 0.95$ and $P$ with $\Phi / \phi \leq 1$. It also follows that under the above conditions as $\Phi / \phi$ exceeds unity all the additional power reaching $D_{4}$ is due to the noise power, leading to decreases of $p$ and $P$. If, on the other hand, the input-beam N.A. is increased until $\phi \approx \phi_{0}$ so that the input signal excites almost all the fiber modes, the signal power per fiber mode is reduced, while that of the noise remains the same, thus leading to a large $R$ as well as to low constant values of $p$ and $P$ that are almost independent of $\phi$. This model is supported by the data of Fig. 3. These were measured by means of one line scan of the TV camera at the center of the phase-conjugate beam, where the background noise was electronically eliminated. It is seen that for a small N.A. $\left[\left(\phi / \phi_{0}\right)^{2}=0.005\right]$ the noise intensity [see Fig. 3(b)] is much smaller than that of the true phaseconjugate component [see Fig. 3(a)] and is distributed more or less uniformly over $\phi$. In the case $\left(\phi / \phi_{0}\right)^{2}=$ 0.69 the noise is distributed over $\phi_{0}$, but most of the noise power is occupied inside $\phi$, resulting in the insensitivity of $p$ and $P$ to $\Phi / \phi$.

In our theoretical model ${ }^{2}$ complete modal equipartition in the fiber has been assumed. In what follows we show that a simple extension of this model that also takes the noise components into account can explain the present results. Using the notation of the previous model, ${ }^{2}$ we can express the phase-conjugate field $\mathbf{E}^{(4)}$ as

$$
\mathbf{E}^{(4)}=r M^{\prime} C M^{*}\left(\mathbf{E}^{(1)}\right)^{*},
$$

where $r$ is the phase-conjugate amplitude reflectivity of the crystal, $M$ and $M^{\prime}$ are the scattering matrices of the fiber in the forward and backward directions, respectively, and $C$ represents the removal of the $y$ polarization by the polarizer P2 (see Fig. 1).

Without loss of generality we shall assume a linearly $x$-polarized incident field $\mathbf{E}^{(1)}$. We shall further assume a lossless transmission through the fiber. Then from $M^{\dagger}=M^{-1}$ (the unitarity condition) and $M^{\prime}=$ $\left(M^{*}\right)^{-1}$ (the time-reversal symmetry) (Ref. 2), we obtain the phase-conjugate intensities of the $i$ th mode for both polarizations:

$$
\left(I_{x}^{(4)}\right)_{i}=|r|^{2} \sum_{l=1}^{N} \sum_{m=1}^{N}\left|\left(M_{x x}\right)_{l i}\right|^{2}\left|\left(M_{x x}\right)_{m i}\right|^{2}\left(I^{(1)}\right)_{i}+\left(N_{x}\right)_{i}
$$

and

$$
\left(I_{y}^{(4)}\right)_{i}=\left(N_{y}\right)_{i},
$$

where $\left.\left(I^{(1)}\right)_{i} \equiv \mid \mathbf{E}^{(1)}\right)\left._{i}\right|^{2}$, and $\left(N_{x}\right)_{i}$ and $\left(N_{y}\right)_{i}$ account for the noise contributions to the phase-conjugate power. As a first approximation we may neglect contributions from interference between modes in the detection plane (which is a far field of the input end of the fiber). Then, taking into account that the measured area is limited by aperture AP2 (see Fig. 1) and that the noise is nearly completely depolarized and uniformly distributed over all the fiber modes, we may express the measured power (i.e., integrated intensities) up to the mode number $M$ as

$$
P_{x}=P_{t}+P_{M} / 2
$$

and

$$
P_{y}=P_{M} / 2,
$$

where $P_{t}=|r|^{2} \sum_{i=1}^{M} \sum_{l=1}^{N} \sum_{m=1}^{N}\left|\left(M_{x x}\right)_{l i}\right|^{2}$ $\left|\left(M_{x x}\right)_{m i}\right|^{2}\left(I^{(1)}\right)_{i}$ is the measured true phase-conjugate beam power and $P_{M}=2 \sum_{i=1}^{M}\left(N_{x}\right)_{i}=2 \sum_{i=1}^{M}\left(N_{y}\right)_{i}$ is the measured noise power. From Eqs. (4) and (5) we have the degree of polarization $P=P_{t} /\left(P_{t}+P_{M}\right)$.

In order to obtain the dependence of $P$ on the inputbeam N.A., we introduce the following modal partition functions for the true phase-conjugate beam power and the noise power, respectively: 


$$
\theta_{i}=\frac{\left(I^{(1)}\right)_{i}}{\sum_{i=1}^{N}\left(I^{(1)}\right)_{i}}
$$

and

$$
\Delta_{i}=\frac{2\left(N_{x}\right)_{i}}{P_{N}}=\frac{2\left(N_{y}\right)_{i}}{P_{N}} .
$$

In Eq. (7) the total noise power $P_{N}\left(\left.\equiv P_{M}\right|_{M=N}\right)$ can be expressed in terms of the total reflected power $P_{0} \equiv$ $\left|\mathbf{E}^{(3)}\right|^{2}$ (injected from the other end of the fiber after phase conjugation by the crystal) minus the total true phase-conjugate beam power $\left.P_{t}\right|_{M=N}$, i.e., $P_{N}=P_{0}-$ $\left.P_{t}\right|_{M=N}$, where $P_{0}$ is given by

$$
\begin{aligned}
P_{0}=|r|^{2}\left[\sum_{l=1}^{N} \sum_{m=1}^{N}\left|\left(M_{x x}\right)_{l m}\right|^{2}\left(I^{(1)}\right)_{m}\right. \\
\left.+\sum_{l=1}^{N} \sum_{k=1}^{N} \sum_{\substack{k^{\prime}=1 \\
\left(k^{\prime} \neq k\right)}}^{N}\left(M_{x x}\right)_{l k}\left(M_{x x}\right)_{l k^{\prime}}^{*}\left(A_{x}^{(1)}\right)_{k}\left(A_{x}^{(1)}\right)_{k^{\prime}}^{*}\right]
\end{aligned}
$$

in which $A_{x}^{(1)}$ is a subcolumn vector representing an amplitude of $\mathbf{E}_{x}^{(1)}$. From the unitarity condition and the experimental observation of the highly depolarized field $\mathbf{E}^{(2)}$ for any input field $\mathbf{E}^{(1)}$ (typically $P_{\text {out }}<$ 0.1 ), we may write

$$
\sum_{l=1}^{N}\left|\left(M_{x x}\right)_{l m}\right|^{2}=1 / 2 \quad \text { for any } m
$$

and neglect the second term in Eq. (8). Using Eqs. (6)-(9), we finally obtain

$$
P=1 /(1+\beta),
$$

where $\beta \equiv P_{M} / P_{t}=\sum_{i=1}^{M} \Delta_{i} / \sum_{i=1}^{M} \theta_{i}$. Note that $\beta=1$ and $P=0.5$ when $M=N$, indicating that the total

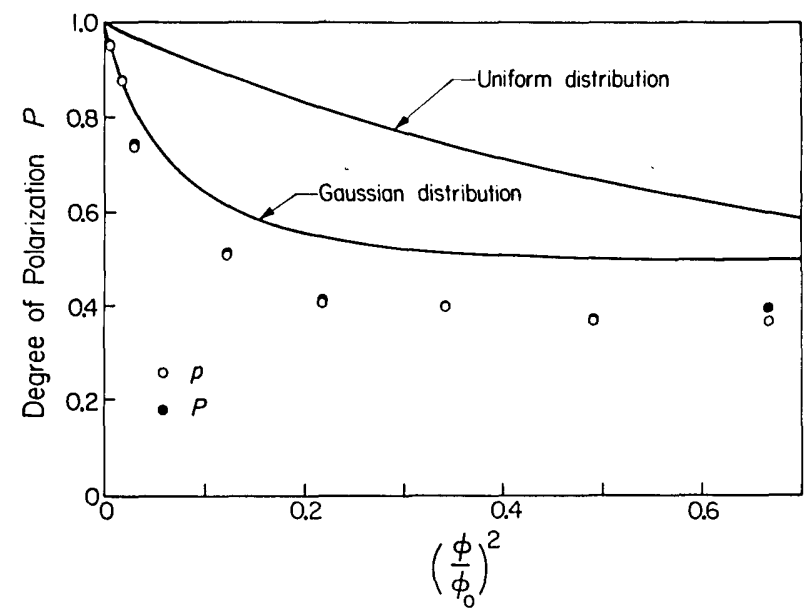

Fig. 4. Theoretical curves of degree of polarization $P$ as a function of $\left(\phi / \phi_{0}\right)^{2}$ for a uniform distribution and Gaussian distributions $\left(\psi / \phi_{0}=0.5\right)$, together with the experimental data shown in Fig. 2: $p(0)$ and $P(\bullet)$. noise power is the same as the total true phase-conjugate beam power and is equally partitioned among two orthogonal polarizations. We also note that in our experiment $\Phi=\phi$, i.e., $\sum_{i=1}^{M} \theta_{i}=1$. For a uniform distribution of the depolarized field, $\sum_{i=1}^{M} \Delta_{i}=M / N=$ (N.A./N.A.fiber $)^{2}=\left(\phi / \phi_{0}\right)^{2}$. For a Gaussian distribution, i.e., $I_{d}\left(r_{i}\right)=I_{0} \exp \left(-2 r_{i}^{2} / \xi^{2}\right)$, where $I_{0}$ is a normalization factor and $\xi=\psi / 2(\psi$ is an effective diameter of the depolarized beam), we may replace the summation by an integration for a large $N$, so that $\sum_{i=1}^{M} \Delta_{i} \approx 1-$ $\exp \left[-2\left(\phi / \phi_{0}\right)^{2} /\left(\psi / \phi_{0}\right)^{2}\right]$.

Figure 4 shows the theoretical curves of the degree of polarization $P$ for the case of the uniform distribution and the Gaussian distribution $\left(\psi / \phi_{0}=0.5\right)$ as a function of $\left(\phi / \phi_{0}\right)^{2}$. The experimental data of Fig. 1 are also reproduced. It is seen that the theoretical curves using the Gaussian distribution are in good agreement with the experiment. The difference between the theory and the experiment for large $\phi$ 's may be due to the leaky and radiation losses of the input field $\mathbf{E}^{(1)}$ from the fiber and the residual polarization of the field $\mathbf{E}^{(2)}$. A detailed theoretical analysis of the fidelity of phase conjugation will be published elsewhere. ${ }^{7}$

In conclusion, we have shown that the fidelity of the true phase conjugation degrades as the input-beam N.A. increases. ${ }^{8}$ This is because the depolarized and diffused noise components in the reflected beam are essentially uniformly distributed over N.A.fiber so that an increase in the signal N.A. simply increases the noise power that intermingles with the signal. A theoretical model to explain the experiment has also been presented. We conclude that to take advantage of the true phase-conjugation feature of the fiber-coupled PCM we need to restrict the angular spread of the input beam so as to excite only a small fraction of the fiber's N.A. (or modes). This may be restrictive for applications involving pictorial information yet permit numerous other applications involving sensors, gyroscopes, and multichannel switching.

This research was supported by the U.S. Air Force Office of Scientific Research and by the U.S. Army Research Office, Durham, North Carolina.

\section{References}

1. K. Kyuma, A. Yariv, and S.-K. Kwong, Appl. Phys. Lett. 49,617 (1986).

2. A. Yariv, Y. Tomita, and K. Kyuma, Opt. Lett. 11, 809 (1986).

3. R. Yahalom, K. Kyuma, and A. Yariv, Appl. Phys. Lett. 50, 792 (1987).

4. S.-K. Kwong, R. Yahalom, K. Kyuma, and A. Yariv, Opt. Lett. 12, 337 (1987).

5. J. Feinberg, Opt. Lett. 7, 486 (1982).

6. M. Born and E. Wolf, Principles of Optics, 5th ed. (Pergamon, New York, 1975), p. 555.

7. Y. Tomita, R. Yahalom, and A. Yariv, "Theory of polarization and spatial information recovery by modal dispersal and phase conjugation," submitted to J. Opt. Soc. Am. B.

8. After the submission of this manuscript we learned that a similar conclusion was independently reported by $\mathrm{I}$. McMichael, P. Yeh, and P. Beckwith, Opt. Lett. 12, 507 (1987). 